\title{
Low Back Pain and Sacroiliitis on Cross-Sectional Abdominal Imaging for Axial Spondyloarthritis Diagnosis in Inflammatory Bowel Diseases
}

\author{
Marine Fauny ${ }^{\mathrm{a}}$ Nicolas Cohen ${ }^{\mathrm{a}}$ Caroline Morizot ${ }^{\mathrm{a}}$ Sophie Leclerc- \\ Jacob $^{a}$ Daniel Wendling ${ }^{b}$ Guillaume Lux ${ }^{c}$ Valérie Laurent ${ }^{c}$ Alain Blum ${ }^{c}$ \\ Patrick Netter $^{d} \quad$ Cédric Baumann ${ }^{e}$ Isabelle Chary-Valckenaere ${ }^{a, d}$ \\ Laurent Peyrin-Biroulet ${ }^{f, g}$ Damien Loeuille ${ }^{a, d}$ \\ aDepartment of Rheumatology, IMoPA, University Hospital of Nancy, Lorraine University, Nancy, France; \\ ${ }^{b}$ Department of Rheumatology, University Hospital of Besancon, Besancon, France; 'Department of Radiology, \\ IMoPA, University Hospital of Nancy, Lorraine University, Nancy, France; Ingénierie Moléculaire et Ingénierie \\ Articulaire (IMoPA), UMR-7365 CNRS, Faculté de Médecine, University of Lorraine and University Hospital of \\ Nancy, Nancy, France; eUnity of Methodology, Data Management and Statistic, University Hospital of Nancy, \\ Nancy, France; fDepartment of Gastroenterology and Hepatology, Inserm NGERE, University Hospital of Nancy, \\ Lorraine University, Nancy, France; IInserm U1256 NGERE, Lorraine University, Vandoeuvre-Les-Nancy, France
}

\section{Keywords}

Sacroiliitis · Imagery · Inflammatory bowel disease ·

Spondyloarthritis

\begin{abstract}
Background: Joint damage is the most frequent extraintestinal manifestation in inflammatory bowel disease (IBD). Aims: The aim of the study was to assess the value of low back pain (LBP) associated with sacroiliitis on abdominal imaging for the diagnosis of spondyloarthritis (SpA) in IBD. Methods: We used a questionnaire assessing rheumatological symptoms for all patients with abdominal computed tomography (CT) and magnetic resonance enterography (MRE). Sacroiliitis was assessed on available CT and MRE. Patients were classified as axial SpA according to the Assessment of SpondyloArthritis International Society criteria. $\boldsymbol{R} \boldsymbol{e}$ -
\end{abstract}

sults: Fifty-one patients completed the questionnaire and performed both exams. LBP was present in 27 patients (52.9\%), and 10 (19.6\%) had an inflammatory component. Sacroiliitis was reported in 12 patients (23.5\%), and 6 of them suffered from LBP. Among the 20 patients referred to the rheumatologist, 11 patients suffered from LBP. One patient was HLA-B27 positive and presented sacroiliitis. For the last 10 patients, none of them had a sacroiliitis, and 2 patients were negative for HLA-B27. Conclusion: An axial SpA has been diagnosed in $11.8 \%$ of IBD patients undergoing crosssectional imaging, whereas one-fifth had inflammatory LBP, and sacroiliitis was observed in one-quarter of them. To optimize the diagnosis of axial SpA, HLA-B27 testing might be required for patients with both $I B D$ and $L B P$, but this will require further investigation before its implementation in routine practice.

(C) 2020 The Author(s)

Published by S. Karger AG, Basel

$\begin{array}{ll}\text { karger@karger.com } & \text { (c) 2020 The Author(s) } \\ \text { Published by S. Karger AG, Basel } & \text { Karger } \\ \text { This article is licensed under the Creative Commons Attribution- } & \text { NonCommercial-NoDerivatives 4.0 International License (CC BY- } \\ \text { Narg-ND) (http://www.karger.com/Services/OpenAccessLicense). } \\ \text { Usage and distribution for commercial purposes as well as any dis- } \\ \text { tribution of modified material requires written permission. }\end{array}$

Marine Fauny

Department of Rheumatology

CHRU Nancy

Rue du Morvan, FR-54500 Vandoeuvre-les-Nancy (France)

m.fauny@chru-nancy.fr 


\section{Introduction}

Joint damage is the most frequent extraintestinal manifestation in patients with inflammatory bowel disease (IBD), including Crohn's disease (CD) and ulcerative colitis (UC), and was first described in 1930 [1]. Rheumatological manifestations in spondyloarthritis (SpA) include peripheral arthritis (oligoarthritis or polyarthritis) and axial involvement based on low back pain (LBP). Axial SpA is defined by LBP, buttock pain, chest pain, and/ or heel pain with an inflammatory or mechanical component, and its prevalence ranges between 2 and $16 \%$ in IBD patients [2-4]. Before 2009, the diagnosis of structural axial SpA was defined by the presence of inflammatory LBP, in the presence of structural sacroiliitis on radiography or computed tomography (CT) scan. In IBD patients, the prevalence of structural sacroiliitis on radiography [5] ranged from 2.9 to $10 \%[6,7]$. In 2009, the Assessment of SpondyloArthritis International Society (ASAS) group published new criteria for the classification of SpA, based not only on the presence of structural sacroiliitis on radiography (like in New York criteria) but also on the presence of inflammatory sacroiliitis on magnetic resonance imaging (MRI) corresponding to the imaging arm [8]. Patients under 45 years with chronic back pain for at least 3 months can be classified as SpA if they have sacroiliitis on radiography or MRI with an SpA feature (inflammatory LBP, arthritis, enthesitis, uveitis, dactylitis, psoriasis, good response under NSAIDs, family history of SpA, HLA-B27+, elevated C-reactive protein [CRP], or IBD) or, without sacroiliitis, if they are HLA-B27+ with at least $2 \mathrm{SpA}$ features. Recently, CT dedicated to the sacroiliac joint (SIJ) and abdominal CT scan permitted also to establish a diagnosis of structural sacroiliitis with the same level of specificity and an excellent sensitivity compared to the radiography [9] and the CT scan of SIJs, considered as the gold standard for the diagnosis of structural sacroiliitis.

The diagnosis and monitoring of IBD has changed with the advent of CT and magnetic resonance enterography (MRE). Abdominal CT and MRE also provide us the possibility to investigate SIJs by diagnosing inflammatory sacroiliitis on MRE [10] and structural sacroiliitis on CT $[9,11]$. We decided to determine for the first time structural and/or inflammatory sacroiliitis prevalence on abdominal $\mathrm{CT}$ and MRE and to characterize the axial musculoskeletal complaints in IBD patients through a dedicated questionnaire.

The main objective of this study was to characterize axial musculoskeletal features and structural and inflam- matory sacroiliitis on abdominal CT and MRE already performed for digestive investigations to diagnose SpA in a population of patients followed up for IBD. Secondary objectives were to evaluate the prevalence of axial SpA according to ASAS criteria (clinical and radiographic arms) and to investigate IBD characteristics associated with axial inflammatory manifestations and structural or inflammatory sacroiliitis.

\section{Methods}

\section{Study Population (Fig. 1)}

Patients with a diagnosis of IBD according to the LennardJones criteria [12] were included if they had performed both MRE and CT abdominal exam. They had to answer a questionnaire about rheumatic symptoms (online suppl. Appendix; for all online suppl. material, see www.karger.com/doi/10.1159/000507930). The exclusion criteria were patients under 18 years, those unable to complete the questionnaire, or those with seriously reduced cognitive capacity.

Between 2004 and 2011, 186 patients performed a MRE during their IBD follow-up, and 78 performed a CT abdominal exam as well. At the time of this study, a questionnaire has been sent to IBD patients for whom both exams have been performed $(n=78)$, and 51 patients have completed all the items of the questionnaire. These 51 IBD patients $(\mathrm{CD}=41, \mathrm{UC}=10)$ underwent CT (thoraco-abdominopelvic [TAP] CT and entero-CT [CTE]) and MRE during their follow-up. The period between imaging modalities did not exceed 2 years.

The digestive localization of IBD was determined according to the Montreal classification [13]. The severity of disease was based on abdominal surgical history excluding dilation of stenosis and ablation of fistulas. IBD was considered endoscopically active in case of the presence of significant mucosal lesions. Information concerning patients addressed to the rheumatologist for musculoskeletal symptoms and HLA-B27 status were systematically searched in clinical records.

\section{Axial and Peripheral Musculoskeletal Symptoms Reported}

through a Dedicated Questionnaire

Largely inspired from ASAS classification criteria, the questionnaire is based on 16 main closed-ended questions about axial and peripheral manifestations. We sent this questionnaire to the patients at the time of this study. Thanks to the questionnaire results, these issues have been reported: (1) axial or pelvic-spinal symptoms, including buttock, heel, low back, and thoracic pain, with inflammatory component defined by morning stiffness $>30$ min and night pain, mechanical component with no night pain and morning stiffness $<30 \mathrm{~min}$, and mixed component with night pain or morning stiffness $>30 \mathrm{~min}$; (2) peripheral symptoms with peripheral pain, with inflammatory, mechanical, or mixed component. Arthritis, enthesitis, and dactylitis diagnosis is based on clinical examination or imagery data, so they have not been presented in this work. 


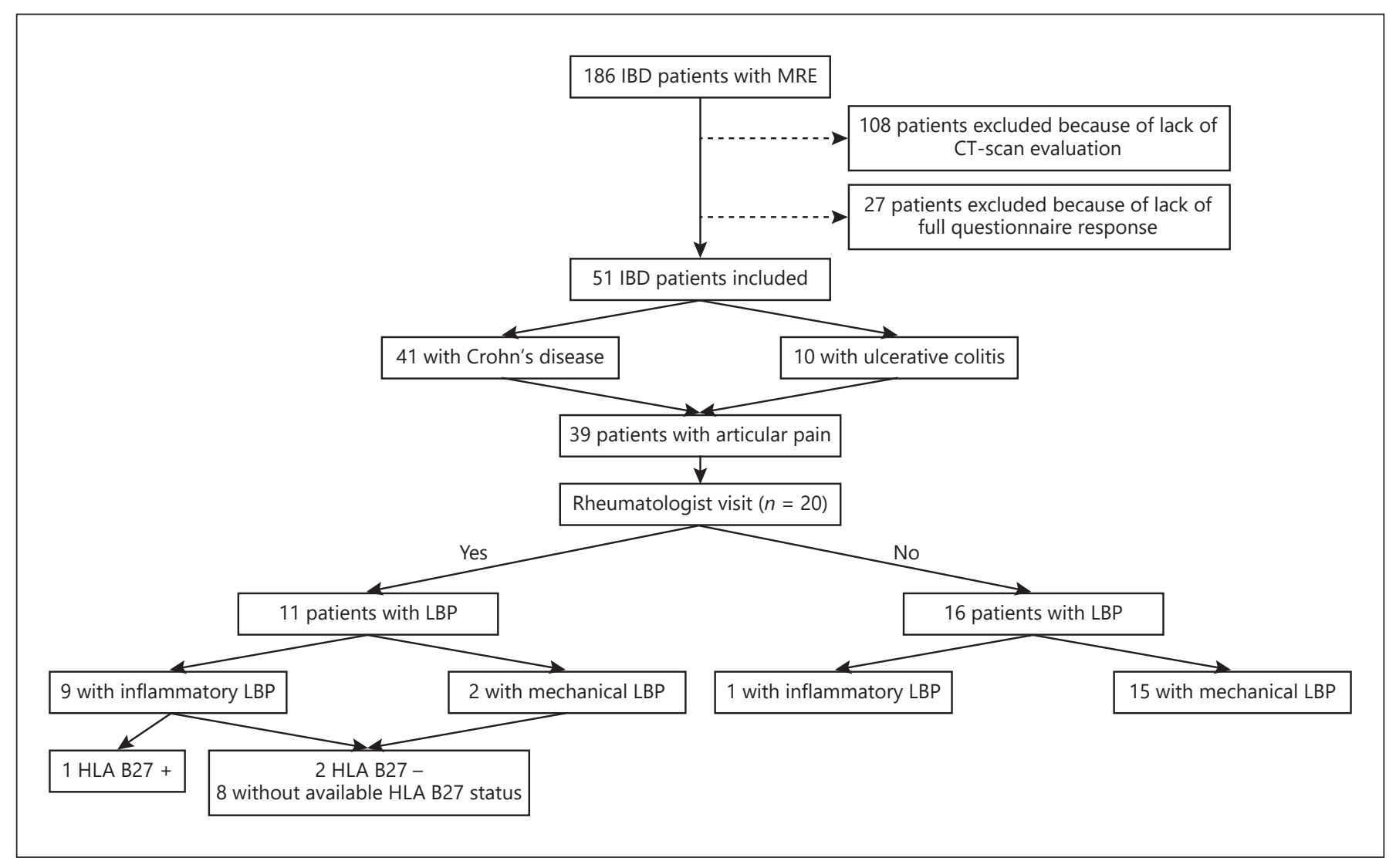

Fig. 1. Patient inclusion flowchart. CT, computed tomography; IBD, inflammatory bowel disease; LBP, low back pain; MRE, magnetic resonance enterography.

\section{Imaging Protocol}

We performed a retrospective study of radiological exams performed between 2004 and 2011.

\section{SIJ Structural Assessment}

- CT technique: CT scans were performed during the follow-up of the patients, to assess the disease extension (in particular parietal or endoluminal extension in CD), comorbidities, treatments side effects, or sometimes for emergency. Forty-five patients performed TAP CT, 6 abdominal CT, and 3 SIJ CT. A LightSpeed General Electric scanner was used for CT. The field of view was the chest, abdominal, and pelvic regions for TAP $\mathrm{CT}$, and the abdominal and pelvic regions for abdominal CT. Imaging was performed on axial contiguous slices. Both imaging methods, with or without injection of contrast agent, allow for assessment of SIJ on about 20 consecutive axial slides in the bone window.

- SIJ CT assessment: Evaluations were performed independently by a rheumatologist (DL) and a radiologist (GL) blinded to the diagnosis and the questionnaire. Sacroiliitis was scored according to the method previously described by Melchior et al. [9]: sclerosis: positive if the depth is $5 \mathrm{~mm}$ (not considered if isolated); erosions: interruption of the sacral or iliac cortical bone if present on at least 2 consecutive slices (grade 2); narrowing or widening of the joint space in the presence of erosions if present on at least 2 consecutive slices compared with the other side (grade 3); partial synostosis: partial bone bridge in the presence of erosions if present on at least 2 consecutive slices (grade 3); and complete synostosis: complete bone bridge present on at least 2 consecutive slices (grade 4). In cases of discordance, the final diagnosis was obtained by consensus.

The diagnosis of structural sacroiliitis on CT is defined by grade 2 for bilateral localization or grade 3 for unilateral localization.

\section{SIJ Inflammatory Assessment}

- MRE technique: Digestive MRE was delimited by the 11th dorsal vertebra and the femoral head, permitting SIJ examination on about 10-12 coronal or axial slices. Two injected T1-weighted sequences with fat saturation were used for the whole population: (1) coronal liver acquisition with volume acceleration sequence: sequence acquisition was made 3 min after contrast agent injection; (2) axial spoiled gradient recalled sequence: sequence acquisition was made $4 \mathrm{~min}$ after contrast agent injection.

- SIJ MRI assessment: Inflammatory sacroiliitis was diagnosed according to ASAS 2009 criteria, in particular the presence of bone marrow edema (BME) in the subchondral or peri-articular compartment of the SIJ on 1 slice for bilateral lesions or on 2 consecutive slices for unilateral lesions [14]. The SIJ were de- 
Table 1. Main clinical and biological characteristics of the population

\begin{tabular}{|c|c|c|c|c|c|}
\hline & $\begin{array}{l}\text { General } \\
\text { population } \\
(n=51)\end{array}$ & \multicolumn{4}{|c|}{ Subgroups of general population } \\
\hline \multicolumn{6}{|l|}{ Demographical data } \\
\hline Smoking & $22(43.1)$ & $11(40.74)$ & $5(50)$ & $5(41.67)$ & $3(50)$ \\
\hline Peripheric arthralgia & $39(76.5)$ & $27(100)$ & $10(100)$ & $10(83.33)$ & $6(100)$ \\
\hline Psoriasis & $7(13.7)$ & $5(18.52)$ & $2(20)$ & $1(8.33)$ & $0(0)$ \\
\hline Family history of psoriasis & $7(13.7)$ & $5(18.52)$ & $2(20)$ & $0(0)$ & $0(0)$ \\
\hline Family history of $\mathrm{SpA}$ & $3(5.9)$ & $3(11.11)$ & $3(30)$ & $0(0)$ & $0(0)$ \\
\hline Uveitis & $2(3.9)$ & $0(0)$ & $0(0)$ & $1(8.33)$ & $0(0)$ \\
\hline \multicolumn{6}{|l|}{ CD Montreal classification } \\
\hline L1 & $11(26.8)$ & $7(25.9)$ & $4(40)$ & $3(25.17)$ & $1(16.67)$ \\
\hline $\mathrm{L} 2$ & $3(7.3)$ & $2(7.41)$ & $1(10)$ & $0(0)$ & $0(0)$ \\
\hline L3 & $27(65.9)$ & $14(51.85)$ & $3(30)$ & $7(58.3)$ & $4(66.67)$ \\
\hline \multicolumn{6}{|l|}{ UC Montreal classification } \\
\hline E1 & $1(10)$ & $0(0)$ & $0(0)$ & $0(0)$ & $0(0)$ \\
\hline E2 & $1(10)$ & $0(0)$ & $0(0)$ & $1(8.33)$ & $1(16.67)$ \\
\hline E3 & $8(80)$ & $4(14.8)$ & $2(20)$ & $1(8.33)$ & $0(0)$ \\
\hline Surgery & $25(49)$ & $14(51.85)$ & $5(50)$ & $8(66.67)$ & $4(66.67)$ \\
\hline Biological inflammation $(\mathrm{CRP}>5)$ at the time of MRI & $46(90.2)$ & $25(92.59)$ & $9(90)$ & $9(75)$ & $5(83.33)$ \\
\hline Bowel flare-up on endoscopy $(n=30)$ & $24(80)$ & $11(40.74)$ & $4(40)$ & $4(33.33)$ & $2(33.33)$ \\
\hline Bowel flare-up on MRI & $33(64.7)$ & $18(66.67)$ & $6(60)$ & $9(75)$ & $5(83.33)$ \\
\hline \multicolumn{6}{|l|}{ Therapy at the time of CT } \\
\hline Corticotherapy & $8(15.7)$ & $5(18.52)$ & $1(10)$ & $0(0)$ & $0(0)$ \\
\hline Immunosuppressor & $10(5.1)$ & $6(22.22)$ & $3(30)$ & $1(8.33)$ & $0(0)$ \\
\hline Biological & $8(15.7)$ & $3(11.11)$ & $2(20)$ & $3(25)$ & $0(0)$ \\
\hline \multicolumn{6}{|l|}{ Therapy at the time of MRI } \\
\hline Corticotherapy & $14(27.5)$ & $8(29.63)$ & $3(30)$ & $3(25)$ & $2(33.33)$ \\
\hline Immunosuppressor & $18(35.3)$ & $10(37.04)$ & $3(30)$ & $3(25)$ & $2(33.33)$ \\
\hline Biological & $23(45.1)$ & $9(33.33)$ & $1(10)$ & $7(58.33)$ & $2(33.33)$ \\
\hline
\end{tabular}

ASAS, Assessment of SpondyloArthritis International Society; CD, Crohn's disease; CRP, C-reactive protein; CT, computed tomography; LBP, low back pain; MRI, magnetic resonance imaging; SpA, spondyloarthritis; UC, ulcerative colitis.

scribed as inflammatory sacroiliitis (sacroiliitis grade, uni- or bilateral) or normal.

\section{Ethics Approval}

Information about the Nancy IBD cohort is reported to the Commission Nationale de l'Informatique et des Libertés, which supervises the implementation of the act regarding data processing, data files, and individual liberties that came into effect on January 6, 1978, and was amended on August 6, 2004, to protect the personal data of individuals. The study was approved by the ethics review board of the Nancy University Hospital (No. 1404720). Patients gave their consent to use their medical data when they were cared for at the university hospital. The study protocol conforms to the ethical guidelines of the 1975 Declaration of Helsinki as reflected in a priori approval by the institution's human research committee. For this type of study, formal consent is not required.

\section{Diagnosis of Axial SpA}

Axial SpA retained on ASAS criteria was defined by the presence of chronic LBP and inflammatory sacroiliitis on digestive MRE and/ or structural sacroiliitis on abdominal CT in the imaging group. Concerning the clinical arm based on the HLA-B27 status, patients should also have chronic LBP and at least 2 other clinical features of SpA according to ASAS criteria (inflammatory LBP, NSAID sensitiveness, spondyloarthropathy family history, psoriasis, uveitis, arthritis, enthesitis, and dactylitis as set out by ASAS definitions [15]). 
Statistical Analysis

Data were analyzed with SAS software for Windows, v9.1 (SAS Institute, Cary, NC, USA). The association between sacroiliitis and the clinical, biological, or radiological parameters of the digestive disease was analyzed by Fisher's exact test or the $\chi^{2}$ test (qualitative variables) and by Wilcoxon's test (quantitative variables) with a $p$ value $<0.05$ as significant.

\section{Results}

\section{Clinical Characteristics of the 51 Patients (Fig. 1)}

Fifty-one IBD patients met the inclusion criteria, with a mean age of 36.8 years and 28 females (54.9\%) (Table 1 ). Patients suffered from CD in $80 \%$ of cases $(n=41)$, and $58.9 \%(n=30)$ of them were in flare according to endoscopic findings. Patients received immunosuppressive treatment and biologic treatment at the time of MRE in $35.2 \%(n=18)$ and $45.1 \%(n=23)$ of cases, respectively. The CRP level was beyond the upper limit of $5 \mathrm{mg} / \mathrm{L}$ for $90.2 \%(n=46)$ of the patients.

\section{Clinical Visit with a Rheumatologist and HLA-B27}

Status (Fig. 1)

Among the 51 IBD patients, 39 (76.5\%) suffered from articular pain, 20 benefited from a rheumatologist visit. From these 20 patients, 11 suffered from LBP, 9 with inflammatory LBP, and 2 with mechanical LBP. Only 1 patient with inflammatory LBP was HLA-B27 positive and presented sacroiliitis on MRE and CT scan. For the last 10 patients with LBP, HLA-B27 was negative in 2 cases, not available for 8 patients, and none presented sacroiliitis on digestive imaging.

\section{Questionnaire Results}

Of the 51 patients, $52.9 \%(n=27)$ presented LBP and $19.6 \%(n=10)$ had an inflammatory component. Axial symptoms were reported in $64.7 \%(n=33)$ of IBD patients: there was an inflammatory component in $39.4 \%$ $(n=13)$, a mechanical component in $21.2 \%(n=7)$, and it was mixed in $39.4 \%$ of them $(n=13)$. The other axial manifestations were in order of frequency: buttock and heel pain in $29.4 \%$ of patients and chest pain in $11.6 \%$ of patients (Table 2). No clinical, biological, or radiological feature was associated with the presence of inflammatory LBP.

\section{Prevalence of Sacroiliitis on Cross-Sectional Abdominal Imaging}

Twelve patients had sacroiliitis on abdominal CT and/ or MRE. The prevalence of sacroiliitis was $23.5 \%$. The
Table 2. Characteristics of rheumatic symptoms of the 51 patients who completed the questionnaire

\begin{tabular}{lrl}
\hline & $N$ & $\%$ \\
\hline Articular pain & 39 & 76.5 \\
Axial manifestations & & \\
$\quad$ Axial pain & 33 & 64.7 \\
Axial inflammatory & 13 & 25.5 \\
Axial mechanical & 7 & 13.7 \\
Axial mixed & 13 & 25.5 \\
Detail of axial manifestations & & \\
LBP & 27 & 52.9 \\
Inflammatory LBP & 10 & 19.6 \\
Buttock pain & 15 & 29.4 \\
$\quad$ Heel pain & 15 & 29.4 \\
Chest pain & 6 & 11.8 \\
Peripheral manifestations & & \\
Peripheral pain & 33 & 64.7 \\
Peripheral inflammatory & 13 & 25.5 \\
Peripheral mechanical & 8 & 15.7 \\
$\quad$ Peripheral mixed & 12 & 23.5 \\
\hline
\end{tabular}

LPB, low back pain.

prevalence of structural sacroiliitis was $9.8 \%(n=5)$ on CT with 2 patients with bilateral grade 2, 2 patients with bilateral grade 3 , and 1 patient with unilateral grade 3 . All patients with structural sacroiliitis suffered from CD. Moreover, 12 patients presented structural SIJ abnormalities but did not respond to the definition of sacroiliitis: 1 patient with grade 2 and grade $1 ; 5$ patients with unilateral grade 2, 3 patients with bilateral grade 1 , and 3 patients with unilateral grade 1 .

The prevalence of inflammatory sacroiliitis was $15.7 \%$ $(n=8)$ on MRE ( 5 unilateral sacroiliitis and 3 bilateral sacroiliitis). Only 1 patient had both structural and inflammatory sacroiliitis (MRE was performed 5 months before CT). Inflammatory bone lesions were detected on 7.2 slices on average. Only 1 patient suffered from UC. No demographic or biological feature was associated with the presence of a structural and/or inflammatory sacroiliitis.

\section{Axial SpA according to ASAS Criteria}

Six patients (11.8\%) had LBP and sacroiliitis on CT $(n=5)$ and/or MRE $(n=1)$ and responded to ASAS criteria for the diagnosis of axial SpA. Of these 6 patients, only one had inflammatory LBP. All these patients had $\mathrm{CD}$. The 2 patients with negative HLA-B27 status did not meet the clinical criteria of axial SpA, while the HLA-B27positive patient presented both inflammatory LBP and sacroiliitis on both digestive imaging methods. 


\section{Discussion}

We conducted for the first time a study where peripheral and axial musculoskeletal involvements in IBD patients were assessed by a specific questionnaire and for whom structural and inflammatory assessments of their SIJ has been evaluated on abdominal CT or digestive MRE performed during the routine follow-up of their IBD. We showed in this cohort of patients undergoing cross-sectional imaging that 6 patients fulfilled the diagnosis of SpA according to ASAS criteria.

Clinical data collection was performed, thanks to a questionnaire inspired by the ASAS criteria of SpA. As demonstrated by Subramaniam et al. [16], a questionnaire permitted to collect precious information concerning axial and peripheral musculoskeletal complaints in IBD patients [16]. We showed that 33 patients had axial manifestations (64.7\%) when all the components are considered: low back, buttock, heel, and chest pain. We showed that half $(52.9 \%)$ of the IBD patients suffered from LBP with an inflammatory component in $19.6 \%$ of cases. In studies where Calin and ESSG (European Spondyloarthropathy Study Group) criteria were used to evaluate patients with IBD, the prevalence of inflammatory LBP in IBD patients ranged from 5 to $30 \%[3,4,7,16-21]$.

Our population is largely composed of patients with $\mathrm{CD}$, in which females are predominant (80.4\%). In a meta-analysis evaluating the prevalence and incidence of peripheral and axial SpA in IBD, the authors demonstrated higher prevalence for axial SpA and peripheral arthritis in CD patients than UC patients [22], but other studies showed no difference $[1,4,23,24]$. In SpA, sex ratio is usually in favor of males in patients with structural sacroiliitis, while prevalence is similar between females and males in patients with inflammatory sacroiliitis on MRI or in HLA-B27-positive patients without sacroiliitis. We also know that structural damages are more severe in men than in women, reducing the possibility to observe structural sacroiliitis in a population where more than $80 \%$ of IBD patients were females [2]. We also confirmed that IBD patients presented also extra digestive features usually reported, such as psoriasis reported in 7 patients (13.7\%) and uveitis in 2 patients, with prevalence similar to those obviously reported in the literature [25-29].

Concerning biological parameters, patients presented high level of CRP in more than $90 \%$ of the cases. Abnormalities concerning this biological parameter are mostly imputed to the IBD since patients are investigated at the time of digestive flare. Moreover, biological inflammation is rarely observed in SpA patients without IBD or extra-

Spondyloarthritis in Inflammatory Bowel Disease Patients articular feature. Only $30 \%$ of SpA patients presented a CRP level over $5 \mathrm{mg} / \mathrm{L}$ [30]. In this study, the HLA-B27 status is rarely available since this genetic marker is fairly researched in IBD patients suffering from chronic LBP. The lack of HLA-B27 status is probably related to the fact that a large majority of IBD patients suffered from mechanical and not inflammatory LBP. In the studies by van Erp et al. [21] and Subramaniam et al. [16], HLA-B27 status was positive in $7(4.6 \%)$ and $15(18.7 \%)$ cases, respectively, in 150 and 80 IBD patients with peripheral and axial involvements. In the literature [3, 23], HLA-B27 was a predisposing factor for inflammatory LBP in IBD patients.

The diagnosis of structural sacroiliitis is usually established on X-ray in clinical practice, while the CT scan of the SIJ is considered as the gold standard method to depict structural damages. However, CT scan of the SIJ use is limited to doubtful diagnosis of sacroiliitis on pelvic radiography in order to limit radiation exposure in a young population. We have previously demonstrated that abdominal CT was able to grade SIJ abnormalities with good sensitivity and excellent specificity when the CT of the SIJ is taken as the referee imaging method [9]. To our knowledge, it was the first study which estimated the prevalence of structural sacroiliitis from an abdominal CT performed in routine practice at $9.8 \%$ in a population of IBD. On radiography, the prevalence of structural sacroiliitis according to the modified New York criteria ranged also from 5.8 to $45 \%$ in IBD patients [14, 15, 23, 25,31 ], but this prevalence is probably underestimated since the abdominal CT presented lower sensitivity than the dedicated SIJ CT [9].

The prevalence of inflammatory sacroiliitis was $15.7 \%$ (8 patients). Inflammatory bone lesions were depicted on 7.2 slices on average, permitting to establish the diagnosis of inflammatory sacroiliitis with a high level of confidence. On MRE, the prevalence of sacroiliitis was probably underestimated due to technical and clinical factors. Technically, we noted the following: (1) a large field of view with lower spatial resolution in comparison to dedicated MRI acquisition for SIJ (spatial resolution of 1.44 $\mathrm{mm}^{3}$ ); (2) acquisitions were performed axially and coronally and not in the preferred anteroposterior semi-coronal plane; (3) a lack of T2 sequences (short tau inversion recovery or $\mathrm{T} 2$ fat saturation), which are considered more sensitive for BME. Clinically, sacroiliitis may be underestimated due to the high percentage of patients given anti$\mathrm{TNF} \alpha$ treatment which produced a $53.6 \%$ reduction in BME in SIJ in patients treated with adalimumab [32].

The prevalence of structural sacroiliitis was lower than inflammatory sacroiliitis ( 9.8 vs. $15.7 \%)$, and only 1 pa- 
tient had at the same time structural and inflammatory sacroiliitis. But we know that structural sacroiliitis succeeded to local inflammation [33]. So, the study of CT and MRE could increase the sensitivity for SpA diagnosis. Retrospective evaluation of digestive radiological exams could reduce the cost and patient irradiation.

One of the main results of this study is the discordance between axial musculoskeletal complaints and structural abnormality, suggesting that both symptoms and joint lesions are dissociated in IBD patients. Similar results have been also reported $[1,11,23,31,34]$ in a large population of SpA patients: sacroiliitis on MRI or CT defined according to the ASAS definition was inconsistently associated to clinical symptoms. van Erp et al. [21] found an association between active IBD and joint/back pain, whereas in our study, no clinicobiological and radiological feature was associated with the presence of inflammatory LBP or with the presence of structural and/or inflammatory sacroiliitis. We did not find any association probably due to a lack of statistical power. Explanations may be advanced: small sample of patients and the imbalance in the number of patients with CD $(80.4 \%)$. The questionnaire provides data about the clinical symptoms, and the combination of this questionnaire and the retrospective evaluation of radiological exams is useful to meet ASAS criteria for SpA diagnosis.

The strengths of our study were to collect clinical information about mechanical or inflammatory axial involvements and also radiological information, thanks to available abdominal CT and digestive MRE to evaluate inflammatory and structural impairments of SIJs. All these exams were performed during the routine followup of IBD. This study was conducted in a large cohort of patients with IBD for whom all data about IBD were systematically recorded [17]. Moreover, CT and MRE evaluations were performed independently by a rheumatologist and a radiologist, and discordances were classified by consensus.

The limits of this study were related to the low number of patients, the heterogeneity of population, the retrospective analysis of some data, and the fact that HLA-B27 status was checked in only 3 patients probably because most IBD patients suffered from mechanical LBP. Many patients with LBP (77.7\%) without sacroiliitis could not be classified according to ASAS criteria. Another limitation is the delay between the radiological exams and the data obtained from the questionnaire. So, these data could be not truly reflecting the clinical condition of patients at the time of radiological exams. The delay between CT and MRE, which did not exceed 2 years, could create a dis- crepancy between these 2 exams and generate underestimation of the prevalence.

In conclusion, half of IBD patients undergoing crosssectional imaging had LBP and one-fifth with an inflammatory component; one-quarter had sacroiliitis on abdominal imaging and half of them had axial SpA according to ASAS criteria. A diagnosis of axial SpA according to ASAS criteria could be made in about $11.8 \%$ of our patients based on the presence of LBP and SIJ abnormalities on imaging exam performed for digestive investigation. The presence of LBP in combination with sacroiliitis on cross-sectional abdominal imaging represents a new and simple strategy to diagnose earlier axial SpA in IBD patients. HLA-B27 status is rarely searched in IBD patients with axial involvement, while it could be helpful to establish a diagnosis of SpA in patients without sacroiliitis. To optimize the diagnosis of axial SpA, HLA-B27 testing may be required for patients with both IBD and LBP (mechanical or inflammatory), but this will require further investigation before its implementation in routine practice.

The combination of a questionnaire about symptoms and the retrospective evaluation of both CT and MRE are useful to meet ASAS criteria and increase the sensitivity for SpA diagnosis. A retrospective evaluation of digestive radiological exams could reduce the cost and the patient irradiation.

\section{Statement of Ethics}

Information about the Nancy IBD cohort is reported to the Commission Nationale de l'Informatique et des Libertés, which supervises the implementation of the act regarding data processing, data files, and individual liberties that came into effect on January 6, 1978, and was amended on August 6, 2004, to protect the personal data of individuals. The study was approved by the ethics review board of the Nancy University Hospital (No. 1404720). Patients gave their consent to use their medical data when they were cared for at the university hospital. The study protocol conforms to the ethical guidelines of the 1975 Declaration of Helsinki as reflected in a priori approval by the institution's human research committee.

\section{Disclosure Statement}

The authors have no conflicts of interest to declare.

\section{Funding Sources}

The authors did not receive any funding. 


\section{Author Contributions}

All of the authors have contributed to the conception or design of the work, or the acquisition, analysis, or interpretation of data for the work; drafted the work or revised it critically for im- portant intellectual content; approved the version to be published; and gave their agreement to be accountable for all aspects of the work in ensuring that questions related to the accuracy or integrity of any part of the work are appropriately investigated and resolved.

\section{References}

1 Salvarani C, Fries W. Clinical features and epidemiology of spondyloarthritides associated with inflammatory bowel disease. World J Gastroenterol. 2009 May 28;15(20):2449-55.

2 Gionchetti P, Calabrese C, Rizzello F. Inflammatory bowel diseases and spondyloarthropathies. J Rheumatol Suppl. 2015;93:21-3.

3 Ossum AM, Palm Ø, Lunder AK, Cvancarova M, Banitalebi H, Negård A, et al. Ankylosing spondylitis and axial spondyloarthritis in patients with long-term inflammatory bowel disease: results from 20 years of follow-up in the IBSEN study. J Crohns Colitis. 2017 Sep 13; 12:96-104.

4 Malaty HM, Lo GH, Hou JK. Characterization and prevalence of spondyloarthritis and peripheral arthritis among patients with inflammatory bowel disease. Clin Exp Gastroenterol. 2017;10:259-63.

5 Van der Linden S, Valkenburg HA, Cats A. Evaluation of diagnostic criteria for ankylosing spondylitis. A proposal for modification of the New York criteria. Arthritis Rheum. 1984; 27(4):361-8.

6 Veloso FT, Carvalho J, Magro F. Immune-related systemic manifestations of inflammatory bowel disease. A prospective study of $792 \mathrm{pa}$ tients. J Clin Gastroenterol. 1996;23(1):29-34.

7 De Vlam K, Mielants H, Cuvelier C, De Keyser F, Veys EM, De Vos M. Spondyloarthropathy is underestimated in inflammatory bowel disease: prevalence and HLA association. J Rheumatol. 2000;27(12):2860-5.

8 Rudwaleit M, Jurik AG, Hermann KG, Landewé R, van der Heijde D, Baraliakos X, et al. Defining active sacroiliitis on magnetic resonance imaging (MRI) for classification of axial spondyloarthritis: a consensual approach by the ASAS/OMERACT MRI group. Ann Rheum Dis. 2009;68(10):1520-7.

9 Melchior J, Azraq Y, Chary-Valckenaere I, Rat AC, Reignac M, Texeira P, et al. Radiography, abdominal CT and MRI compared with sacroiliac joint CT in diagnosis of structural sacroiliitis. Eur J Radiol. 2017 Oct;95:169-76.

10 Leclerc-Jacob S, Lux G, Rat AC, Laurent V, Blum A, Chary-Valckenaere I, et al. The prevalence of inflammatory sacroiliitis assessed on magnetic resonance imaging of inflammatory bowel disease: a retrospective study performed on 186 patients. Aliment Pharmacol Ther. 2014 May;39(9):957-62.

11 Scott WW Jr, Fishman EK, Kuhlman JE, Caskey CI, O'Brien JJ, Walia GS, et al. Computed tomography evaluation of the sacroiliac joints in Crohn disease. Radiologic/clinical correlation. Skeletal Radiol. 1990;19(3):207-10.
12 Lennard-Jones JE. Classification of inflammatory bowel disease. Scand J Gastroenterol. 1989;24(Suppl 170):2-6.

13 Silverberg MS, Satsangi J, Ahmad T, Arnott ID, Bernstein CN, Brant SR, et al. Toward an integrated clinical, molecular and serological classification of inflammatory bowel disease: report of a Working Party of the 2005 Montreal World Congress of Gastroenterology. Can J Gastroenterol. 2005;19(Suppl A):5A-36A.

14 Steer S, Jones H, Hibbert J, Kondeatis E, Vaughan R, Sanderson J, et al. Low back pain, sacroiliitis, and the relationship with HLA-B27 in Crohn's disease. J Rheumatol. 2003;30(3): $518-22$.

15 Akkoc N, Khan MA. ASAS classification criteria for axial spondyloarthritis: time to modify. Clin Rheumatol. 2016 Jun;35(6):1415-23.

16 Subramaniam K, Tymms K, Shadbolt B, Pavli P. Spondyloarthropathy in inflammatory bowel disease patients on TNF inhibitors. Intern Med J. 2015;45(11):1154-60.

17 Oussalah A, Laurent V, Bruot O, Bressenot A, Bigard MA, Régent D, et al. Diffusion-weighted magnetic resonance without bowel preparation for detecting colonic inflammation in inflammatory bowel disease. Gut. 2010;59(8):1056-65.

18 Orchard TR, Wordsworth BP, Jewell DP. Peripheral arthropathies in inflammatory bowel disease: their articular distribution and natural history. Gut. Mar 1998;42(3):387-91.

19 Salvarani C, Vlachonikolis IG, van der Heijde DM, Fornaciari G, Macchioni P, Beltrami M, et al. Musculoskeletal manifestations in a population-based cohort of inflammatory bowel disease patients. Scand J Gastroenterol. 2001 Dec 12;36(12):1307-13.

20 Dougados M, van der Linden S, Juhlin R, Huitfeldt B, Amor B, Calin A, et al. The European Spondylarthropathy Study Group preliminary criteria for the classification of spondylarthropathy. Arthritis Rheum. 1991 Oct 10; 34(10):1218-27.

21 van Erp SJ, Brakenhoff LK, van Gaalen FA, van den Berg R, Fidder HH, Verspaget HW, et al. Classifying back pain and peripheral joint complaints in inflammatory bowel disease patients: a prospective longitudinal follow-up study. J Crohns Colitis. 2015;10(2):166-75.

22 Karreman MC, Luime JJ, Hazes JMW, Weel AEAM. The prevalence and incidence of axial and peripheral spondyloarthritis in inflammatory bowel disease: a systematic review and meta-analysis. J Crohns Colitis. 2016;11(5): 631-42.

23 Turkcapar N, Toruner M, Soykan I, Aydintug OT, Cetinkaya H, Duzgun N, et al. The preva- lence of extraintestinal manifestations and HLA association in patients with inflammatory bowel disease. Rheumatol Int. 2006;26(7): 663-8.

24 Stolwijk C, Pierik M, Landewé R, Masclee A, van Tubergen A. Prevalence of self-reported spondyloarthritis features in a cohort of patients with inflammatory bowel disease. Can J Gastroenterol. 2013;27(4):199-205.

25 Karmiris K, Avgerinos A, Tavernaraki A, Zeglinas C, Karatzas P, Koukouratos T, et al. Prevalence and characteristics of extra-intestinal manifestations in a large cohort of Greek patients with inflammatory bowel disease. J Crohns Colitis. 2016;10(4):429-36.

26 De Simone C, Sollena P, Coco V, Caldarola G. Psoriasis and erythema nodosum: two comorbidities of inflammatory bowel diseases. G Ital Dermatol Venereol. 2013;148(2):175-84.

27 Mintz R, Feller ER, Bahr RL, Shah SA. Ocular manifestations of inflammatory bowel disease. Inflamm Bowel Dis. 2004;10(2):135-9.

28 Salmon JF, Wright JP, Murray AD. Ocular inflammation in Crohn's disease. Ophthalmology. 1991;98(4):480-4.

29 Krachmer J, Mannis M, Holland E. Cornea. 3rd ed. Elsevier; 2011.

30 Hermann J, Giessauf H, Schaffler G, Ofner P, Graninger W. Early spondyloarthritis: usefulness of clinical screening. Rheumatology (Oxford). 2009 Jul;48(7):812-6.

31 McEniff N, Eustace S, McCarthy C, O’Malley M, O’Morain CA, Hamilton S. Asymptomatic sacroiliitis in inflammatory bowel disease assessment by computed tomography. Clin Imaging. 1995;19(4):258-62.

32 Lambert RG, Salonen D, Rahman P, Inman RD, Wong RL, Einstein SG, et al. Adalimumab significantly reduces both spinal and sacroiliac joint inflammation in patients with ankylosing spondylitis: a multicenter, randomized, double-blind, placebo-controlled study. Arthritis Rheum. 2007;56(12):4005-14.

33 Bennett AN, McGonagle D, O'Connor P, Hensor EM, Sivera F, Coates LC, et al. Severity of baseline magnetic resonance imaging-evident sacroiliitis and HLA-B27 status in early inflammatory back pain predict radiographically evident ankylosing spondylitis at eight years. Arthritis Rheum. 2008;58(11):3413-8.

34 Arnbak B, Grethe Jurik A, Hørslev-Petersen K, Hendricks O, Hermansen LT, Loft AG, et al. Associations between spondyloarthritis features and magnetic resonance imaging findings: a cross-sectional analysis of 1,020 patients with persistent low back pain. Arthritis Rheumatol. 2016;68(4):892-900 